\title{
Quadratus lumborum block versus transversus abdominis plane block for postoperative analgesia in patients undergoing abdominal surgeries: $A$ systematic review and meta-analysis of randomized controlled trials
}

\section{Xiancun Liu}

Jilin University Norman Bethune Health Science Center

\section{Tingting Song}

Jilin University Norman Bethune Health Science Center

\section{Xuejiao Chen}

Beijing University of Chinese Medicine

Jingjing Zhang

First Affiliated Hospital to Changchun University of Chinese Medicine

\section{Conghui Shan}

Jilin University Norman Bethune Health Science Center

\section{Liangying Chang}

Jilin University Norman Bethune Health Science Center

Haiyang Xu ( $\sim$ haiyang1975@163.com )

Jilin University Norman Bethune Health Science Center

\section{Research article}

Keywords: pain scores, abdominal surgery, quadrayus lumborum (QL) block, transversus abdominis plane (TAP) block, meta-analysis

Posted Date: November 18th, 2019

DOI: https://doi.org/10.21203/rs.2.17435/v1

License: (c) (1) This work is licensed under a Creative Commons Attribution 4.0 International License. Read Full License 


\section{Abstract}

Background Abdominal surgery is common and associated with severe postoperative pain. Transverse abdominal plane (TAP) block is considered an effective means for pain control in such cases. Quadratus lumborum (QL) block is another option for the management of postoperative pain. The aim of this study was to conduct a meta-analysis and thereby evaluate the efficacy and safety of QL block and TAP block for pain management after abdominal surgery.

Methods We comprehensively searched PubMed, Embase, EBSCO, the Cochrane Library, Web of Science and CNKI for randomized controlled trials (RCTs) that compared QL block and TAP block for pain management in patients undergoing abdominal surgery. All of the data were screened and evaluated by two researchers. RevMan5.3 was applied to perform the meta-analysis.

Results A total of 8 RCTs involving564 patients were included. The meta-analysis showed statistically significant differences between the two groups with respect to postoperative pain scores at 2 hours (standardized mean difference [Std.MD]=-1.76; 95\% confidence interval [CI]=-2.63 to $-0.89 ; \mathrm{p}<.001$ ), 4 hours (Std.MD $=-0.77 ; 95 \% \mathrm{Cl}=-1.36$ to $-0.18 ; \mathrm{p}=.01$ ), 6 hours (Std.MD $=-1.24 ; 95 \% \mathrm{Cl}=-2.31$ to -0.17 ; $\mathrm{p}=.02$ ), 12 hours (Std.MD $=-0.70 ; 95 \% \mathrm{Cl}=-1.27$ to $-0.13 ; \mathrm{p}=.02$ ) and 24 hours (Std.MD $=-0.65 ; 95 \% \mathrm{Cl}=-1.29$ to $-0.02 ; p=.04)$; postoperative opioid consumption at $24 \mathrm{~h}($ Std. $\mathrm{MD}=-1.39 ; 95 \% \mathrm{Cl}=-1.83$ to $-0.95 ; \mathrm{p}<.001)$; and duration of postoperative analgesia (Std.MD=2.30;95\% $\mathrm{Cl}=1.85$ to $2.75 ; \mathrm{p}<.001$ ). There was no statistically significant difference between the two groups regarding postoperative nausea and vomiting (PONV) incidence (RR=0.55; $95 \% \mathrm{Cl}=0.27$ to $1.14 ; \mathrm{p}=.11$ ).

Conclusion QL block provides better pain management with less opioid consumption than TAP block after abdominal surgery. In addition, there are no differences between TAP block and QL block with respect to PONV.

\section{Background}

There are many kinds of abdominal surgeries, including (but not limited to) colorectal resection, appendectomy, cesarean section, hysterectomy, and laparoscopic cholecystectomy[1]. Postoperative pain is severe in patients undergoing abdominal surgery, and severe pain will not only affect the rapid recovery of patients but also produce a series of pathophysiological reactions[1]. Therefore, it is very important for perioperative patients to have a safe and effective pain management model. The classic postoperative analgesia methods after abdominal surgery are patient-controlled intravenous analgesia (PCIA) and patient-controlled epidural analgesia (PCEA), but both treatments have many adverse effects[2, 3].Opioids can cause nausea and vomiting, respiratory inhibition, lethargy and other complications in self-controlled intravenous analgesia[3]. Epidural analgesia, though it is a better analgesia mode than intravenous analgesia for accelerating the recovery of gastrointestinal function, may still cause common complications, such as motor block, urinary retention and hypotension[4]. Recently, with the rise in enhanced recovery after surgery,nerve block has become the key link in multimodal analgesic regimes[5]. 
As an effective part of multimode analgesia, quadratus lumborum (QL) block and transversus abdominis plane (TAP) block are mainly used for combined anesthesia and postoperative analgesia in abdominal surgery[1]. Compared with TAP block, which is a more mature regional block, QL block was proposed later; however, its embryonic form can be found in many previous articles, and it shows a better blocking effect. At present, many meta-analyses [6-9]have shown that TAP block and QL block can reduce the score of postoperative pain and reduce the dosage of opioids and their related side effects. However, there is no agreement on which type of block is more effective in the management of pain after abdominal surgery. Some studies[10-12] have shown that compared with TAP block, QL block is more effective in postoperative analgesia and can prolong the analgesic time of patients. However, some scholars [13] have confirmed that the analgesic effects of the two treatments are the same in the postoperative period, and there is no difference in the incidence of postoperative adverse reactions. Taken together, QL block and TAP block are still controversial in terms of pain management after abdominal surgery.

Therefore, the purpose of this study was to evaluate, in the form of a meta-analysis, whether QL block or TAP block can be better used for postoperative pain management and reduce the incidence of adverse reactions after abdominal surgery.

\section{Methods}

The study was a meta-analysis, and ethical approval was not required. This review and meta-analysis was reported on the basis of Preferred Reporting Items for Systematic Review and Meta-Analyses (PRISMA).

\section{Search strategy}

We searched the following databases (the time limit is from the establishment of the database to September 2019): PubMed, Embase, EBSCO, the Cochrane Library, Web of Science and CNKI to find randomized controlled trials (RCTs) comparing QL block and TAP block for analgesia after abdominal surgery. The reference lists within these publications were also investigated to identify other hidden authorized trials. No restraints were set on the publication language. The search terms included "quadratus lumborum block", "QL block”, "transversus abdominis plane block”, "TAP block”, "abdominal surgery", "abdominal wall", "abdominal muscles", "pain management", "postoperative pain control", and "postoperative pain management".

\section{Inclusion criteria and study selection}

(1) Population: patients undergoing abdominal surgery; (2) Study design: RCTs; (3) Interventions: QL block; (4) Comparison: TAP block; (5) Primary outcomes: postoperative pain scores and postoperative opioid consumption; and(6) Secondary outcomes: PONV incidence and postoperative analgesia duration. 
Two reviewers searched for and selected studies according to the abovementioned strategy. Specific process: (1) Retrieved references were deduplicated using Endnote software; (2) Screening was initially performed by reading the titles and abstracts; (3) The full texts of the initially screened articles were read, followed by selecting the eligible documents and conducting a risk assessment for bias; (4) The third searcher made the final decision in case of any disagreement with respect to studies that were included.

\section{Data extraction}

Two investigators extracted data from each included study, including basic information (author name, number of cases, gender, age, type of surgery, published year) of the participants, primary outcomes (pain scores and opioid consumption) and secondary outcomes (postoperative analgesia duration and PONV incidence).

\section{Assessment of methodological quality}

The methodological quality of each RCT was evaluated by two investigators, who depended on the Cochrane Handbook, and the third searcher made the final decision in case of any disagreement. The assessment involved random sequence generation, allocation scheme hiding, blinding, accuracy of data results, and freedom from selective reporting and other bias. The quality of the outcomes in the metaanalysis was evaluated by the Grading of Recommendations Assessment, Development and Evaluation (GRADE) (Table 2).

\section{Statistical analysis}

The statistical analysis was conducted using RevMan 5.3. We performed a heterogeneity test on the included studies and calculated the statistics. When $\mathrm{I}^{2}<0.5$, or $\mathrm{p}>0.1$, few heterogeneity was indicated, and a fixed-effects model was applied. Otherwise, a random-effects model was used to analyze the causes of heterogeneity. The continuous outcomes were calculated as the standardized mean difference (Std.MD) with the $95 \%$ confidence interval $(\mathrm{Cl})$. Dichotomous outcomes were measured as relative risk (RR) with the $95 \% \mathrm{Cl}$. Due to the limited number $(<10)$ of included studies, publication bias was not evaluated.

\section{Results}

\section{Literature search and study characteristics}

A total of 135 relevant studies were initially detected, and 8 studies[11-18] were eventually included,comprising 564 patients. The process and results of the literature screening are shown in Fig. 1. The basic features of the 8 RCTs in the meta-analysis are generalized in Table 1. 


\section{Risk of bias}

The Cochrane Handbook for Systematic Review of Interventions was used to evaluate the risk of bias of the RCTs. Five studies[11, 13,15-17] employed the surefire means of using a random number table,two studies $[14,18]$ adopted the method of computer generation of random numbers, and one study[12] applied the closed envelope technique.All studies described the allocation concealment. One study[17] did not mention the blind method employed for the subjects. The researchers used the blind method as well as the rest. Three studies[14,16,18]made use of blinding for outcome measurements, and five other studiesdid not. In addition, all studies reported the completion of the trial and no withdrawals. Only one study[18] reported other high biases. (See Fig. 2 and Fig. 3.)

\section{Outcomes of the meta-analysis}

\section{Postoperative pain scores at 2 hours}

Three studies[11, 12,16]with 180 patients showed pain scores at 2 hours after abdominal surgery. A randomized-effects model was used because significant heterogeneity was found among the studies ( $\mathbb{\nabla}^{2}$ $=0.83, p<.10)$. There was a significant difference in postoperative pain scores at 2 hours between the 2 groups (Std. $M D=-1.76 ; 95 \% \mathrm{Cl}=-2.63$ to $-0.89 ;$ p <.001; Fig. 4 .

\section{Postoperative pain scores at $\mathbf{4}$ hours}

Six studies[11-13,15-17] with 397 patients demonstrated pain scores at 4 hours after abdominal surgery. A random-effects model was applied because significant heterogeneity was found among the studies $\left(\nabla^{2}=0.87, p<.10\right)$. There were significant differences in postoperative pain scores at 4 hours between the 2 groups (Std.MD $=-0.77 ; 95 \% \mathrm{Cl}=-1.36$ to $-0.18 ; \mathrm{p}=.01 ;$;ig. 4 ).

\section{Postoperative pain scores at 6 hours}

Four studies[11, 12, 16, 18]with 287 patients demonstrated pain scores at 6 hours after abdominal surgery. A random-effects model was applied because significant heterogeneity was found among the studies $\left(\mathbb{\nabla}^{2}=0.94, p<.10\right)$. There was no significant difference in postoperative pain scores at 6 hours between the 2 groups (Std.MD $=-1.24 ; 95 \% \mathrm{Cl}=-2.31$ to $-0.17 ; \mathrm{p}=.02 ;$ Fig. 4 ).

\section{Postoperative pain scores at 12 hours}

Seven studies[11-13, 15-18] with 504 patients showed pain scores at 12 hours after abdominal surgery. A random-effects model was used because significant heterogeneity was found among the studies $\left(I^{2}=\right.$ 
$0.89, \mathrm{p}<.10)$. There was a significant difference in postoperative pain scores at 12 hours between the 2 groups (Std.MD $=-0.70 ; 95 \% \mathrm{Cl}=-1.27$ to $-0.13 ; \mathrm{p}=.02$;Fig. 4 ).

\section{Postoperative pain scores at 24 hours}

Seven studies[11-13,15-18] with 504 patients showed pain scores at 24 hours after abdominal surgery. A randomized-effects model was adopted because significant heterogeneity was found among the studies $\left(I^{2}=0.91, p<.10\right)$. There was no significant difference in postoperative pain scores at 24 hours between the 2 groups (Std.MD $=-0.65 ; 95 \% \mathrm{Cl}=-1.29$ to $-0.02 ; \mathrm{p}=.04 ; \mathrm{Fig} .4$ ).

\section{Postoperative opioid consumption at 24 hours}

Five studies[11-14,16-17]with 363 patients showed opioid consumption at 24 hours after abdominal surgery. A random-effects model was used because significant heterogeneity was found among the studies $\left(I^{2}=0.72, p<.10\right)$. There was a significant difference in postoperative opioid consumption at 24 hours between the 2 groups $(S t d . M D=-1.39 ; 95 \% \mathrm{Cl}=-1.83$ to $-0.95 ; \mathrm{p}<.001 ; \mathrm{Fig}$. $5 \rrbracket$.

\section{Duration of postoperative analgesia}

Two studies[11,16]with 130 patients illustrated the analgesia duration after abdominal surgery. A fixedeffects model was adopted because significant heterogeneity was found among the studies $\left(\mathbb{Z}^{2}=0\right.$, p>.10). There was a significant difference in postoperative analgesia duration between the 2 groups(Std.MD = 2.30; 95\% Cl 95\% Cl = 1.85 to $2.75 ;$ p<.001; Fig. 6).

\section{Postoperative nausea and vomiting}

Four studies[14-15,17-18]with 304 patients showed the incidence of PONV. A fixed-effects model was used because no significant heterogeneity was found among the studies $\left(\nabla^{2}=0, p>.10\right)$. There was a significant difference in PONV between the 2 groups ( $R R=0.55 ; 95 \% \mathrm{Cl}=0.27$ to $1.14 ; \mathrm{p}=0.11 ; \mathrm{Fig} .7)$.

\section{Discussion}

Based on 8 randomized controlled trials, the results of the present meta-analysis revealed that postoperative pain scores at $2 \varangle 4 \varangle 6 \bigotimes 12$ and 24 hours were significantly lower in the QL group than in the TAP group. The postoperative opioid consumption was lower in the QL group than in the TAP group. The duration of postoperative analgesia was longer in the QL group than in the TAP group. In addition, there were no differences in PONV.

In the UK, approximately 700,000 people undergo abdominal surgery every year[19]. Patients will experience severe pain, which will lead to a series of complications. Due to pain and discomfort, patients 
do not dare to cough and cannot carry out normal activities, resulting in respiratory complications that may lead to pulmonary infection[4, 20]. If the symptoms are severe, patients may have postoperative delirium, myocardial ischemia and other serious complications. If the pain cannot be eliminated in time, acute pain will be transformed into chronic pain, which will make the patient uneasy, affect wound healing, reduce the quality of life of the patient, and prolong his or her hospitalization time[21, 22]. Therefore, good postoperative analgesia has important clinical significance. In recent years, regional block, as a key link in multimode analgesia, has been increasingly widely used in postoperative analgesia after abdominal surgery. TAP block and QL block are a part of this treatment category $[5,23]$. Thus, the prospect of analgesia after abdominal surgery is becoming increasingly brighter.

Transverse abdominal muscle plane block is a nerve block technique under body surface localization that was described by Rafi for the first time in 2001[24]. Through the Petit triangle (that is, the lower lumbar triangle: the outer boundary is the posterior edge of the abdominal external oblique muscle, the inner boundary is the leading edge of the latissimus dorsi muscle, and the lower boundary is the iliac crest). The TAP is a potential anatomical space between the transverse abdominal muscle and the medial oblique muscle[25]. It originates from the thoracolumbar nerve from the T6 to L1 segment of the spinal nerve root and innervates the abdominal wall, providing anterolateral sensation. The injection of local anesthetics into this space can block nerve afferents and provide good analgesia for the anterolateral abdominal wall[26]. However, due to the narrow block range of abdominal transverse muscle plane block, it is often limited to the postoperative analgesia for lower abdominal surgery, and the application of postoperative analgesia for upper abdominal surgery is limited. As a new technique of abdominal trunk block, QL block was first proposed by Blanco U6I in 2007, who injected local anesthetics into the fascia around the psoas quadratus muscle to block the innervation of the anterior abdominal wall[27]. The block level is high (T7-L1), which can provide postoperative analgesia for both upper and lower abdominal surgery. The key to the analgesic effect of QL block is the thoracolumbar fascia (TLF). The TLF is a complex tubular structure formed by connective tissue. Local anesthetics can spread in the TLF and spread to the paravertebral space to form an indirect paraspinal block[28, 29]. Therefore, it has a certain effect on visceral pain and abdominal incision pain. Additional studies have shown that two different trunk blocks have good analgesic effects in the management of pain after abdominal surgery[7, 10, 27]. Fusco $P$ et al [30]confirmed the analgesic effect of TAP block after cesarean section. At the same time, Blanco et al [14]conducted a randomized controlled trial of 76 patients after cesarean section to compare the effects of pain management after QL block and TAP block. The results showed that TAP block was more able to reduce postoperative morphine requirements. However, there was no significant difference in postoperative pain score between the two groups. Apart from clinical trials, additional meta-analyses have confirmed the feasibility of TAP block and QL block in analgesia after abdominal surgery.

Previous studies have reported the effectiveness and safety of QL block or TAP block in postoperative pain management after abdominal surgery. However, it is not yet clear which treatment is better. Zhu et al[15] found that the difference in VAS scores between QL block and TAP block at 4 hours and 8 hours after surgery had no statistical significance, and the resting and motor scores at 12 hours and 24 hours after surgery in the QL block group were lower than those in the TAP block group. However, Oksuz et 
al[12] reported that the QL block provided more valid analgesic relief. Comparing the number of patients who needed analgesia in the first 24 hours, as well as the scores of 30 min and 1, 2, 4, 6, 12, and 24 hour(s), QL block was observed to be significantly superior to TAP block. At the same time, Kumar's study[16] demonstrated that the pain scores of the patients in the QL block group were lower than those in the TAP block group at 2, 4, 8, 12 and 24 hours following lower abdominal surgeries.

In contrast to the above studies, we systematically evaluated the analgesic effects and adverse reactions of QL block and TAP block in order to obtain a better regional block technique for pain management after abdominal surgery. The results of our meta-analysis by integrating 8 RCTs indicated that QL block was superior to TAP block with respect to the analgesic effect at $2 \varangle 4 \varangle 6 \varangle 12$ and 24 hours after surgery. Overall, the present study suggests that the effect of QL block is better than that of TAP block in the management of pain after abdominal surgery in early periods. We found that QL block is superior to TAP block in reducing postoperative opioid requirements and that the pain control lasts longer after QL block, which is consistent with the findings of Blanco et al. The reason may be that the TLF is formed by the arrangement of the anterior, middle and posterior layers. After the posterior layer and the middle layer meet at the lateral edge of the vertical spinal muscle, they converge with the anterior layer at the lateral edge of the lumbar quadratus muscle to form the aponeurosis starting point of the transverse abdomen muscle. When QL block is performed, the local anesthetics can spread not only within the TLF but also to the abdominal transverse muscle plane and paraspinal space, which is similar to the effect of paravertebral nerve block[31]. The TLF has receptors that can regulate autonomic nerve function and pain mechanisms. Local anesthetics applied to the QL block some sympathetic nerves and thereby achieve a better blocking effect. There was no significant difference in the incidence of PONV between the two groups. The reasons may be related to the different methods of anesthesia but may also stem from the sample size; therefore a large number of consistent clinical trials are still needed.

Regarding the sensitivity analysis, there was still significant heterogeneity when performing the analysis by via omitting one study in turn or subgroup analysis The main reasons for heterogeneity include the following: (1) Five RCTsoriginated in Asia, and the patient sample of one of the RCT studies was limited to children. There may be relevant differences in the analytical results of the integrated data. (2) The types of surgery varied, with some operations being cesarean sections; some, total abdominal hysterectomies; and some, appendectomies. Consequently, the degree of postoperative pain varies among patients undergoing abdominal surgery. (3)The anesthetic drugs and concentrations used in the RCT group were different. The drugs used in the 4 RCTs were bupivacaine, the concentrations of which were $0.125 \%, 0.2 \%$ and $0.25 \%$. The concentrations of ropivacaine used in the other 4 RCTs were $0.25 \%$ and $0.375 \%$. (4)Three RCTs used subarachnoid anesthesia, and five RCTs employed general anesthesia.

The limitations of this meta-analysis are as follows: in the data extraction, some observation indexes in the literature were only reported as the mean and median or in the form of graphics and text; thus, these results could not be included in the analysis, which may have excluded some high-quality literature. Furthermore, there was no explicit mention of the optimal drug type and concentration of the two trunk plane blocks, which need to be further studied to arrive at a satisfactory approach; during the data 
collection process, the author was sincerely asked for the original data by e-mail, but no response was received.

\section{Conclusions}

Compared with TAP block, QL block provides better pain management with less opioid consumption after abdominal surgery.. However, further large randomised controlled trials are required to confirm these findings.

\section{Declarations}

Abbreviations: $\mathrm{Cl}=$ confidence interval $\mathrm{SMD}=$ standard mean difference $\mathrm{PONV}=$ postoperative nausea and vomiting RCTs=randomized controlled trails RR=relative risk VAS: Visual analogue scale

\section{Ethics approval and consent to participate}

Not applicable.

\section{Consent for publication}

Not applicable.

\section{Availability of data and materials}

The datasets used and/or analysed during the current study are available

from the corresponding author on reasonable request.

\section{Competing interests}

The authors declare that they have no competing interests.

\section{Funding}

No funding. 


\section{Author contributions}

$\mathrm{HX}, \mathrm{JZ}$ and LC designed and conceived the study, performed the statistical

analysis, and drafted the manuscript. XC and CS participated in the

interpretation of data and drafting of the manuscript. XL and TS

participated in the study design and helped to draft the manuscript.

All authors read and approved the final manuscript.

\section{Acknowledgements}

Not Applicable.

\section{Author details}

${ }^{1}$ Department of Anesthesiology, Jilin University First Hospital, Changchun, Jilin, China. ${ }^{2}$ Department of Anesthesiology, China-Japan Friendship Hospital, Beijing, China.

\section{References}

1.Borglum J, Gogenur I, Bendtsen TF: Abdominal wall blocks in adults. Current opinion in anaesthesiology 2016, 29(5):638-643.

2.Wu CL, Cohen SR, Richman JM, Rowlingson AJ, Courpas GE, Cheung K, Lin EE, Liu SS: Efficacy of postoperative patient-controlled and continuous infusion epidural analgesia versus intravenous patientcontrolled analgesia with opioids: a meta-analysis. Anesthesiology 2005, 103(5):1079-1088; quiz 1109 1010.

3.Cho JS, Kim HI, Lee KY, Son T, Bai SJ, Choi H, Yoo YC: Comparison of the effects of patient-controlled epidural and intravenous analgesia on postoperative bowel function after laparoscopic gastrectomy: a prospective randomized study. Surgical endoscopy 2017, 31(11):4688-4696.

4.Salicath $\mathrm{JH}$, Yeoh EC, Bennett $\mathrm{MH}$ : Epidural analgesia versus patient-controlled intravenous analgesia for pain following intra-abdominal surgery in adults. The Cochrane database of systematic reviews 2018, 8:Cd010434. 
5.Wick EC, Grant MC, Wu CL: Postoperative Multimodal Analgesia Pain Management With Nonopioid Analgesics and Techniques: A Review. JAMA surgery 2017, 152(7):691-697.

6.Liu L, Xie YH, Zhang W, Chai XQ: Effect of Transversus Abdominis Plane Block on Postoperative Pain after Colorectal Surgery: A Meta-Analysis of Randomized Controlled Trials. Medical principles and practice $2018,27(2): 158-165$.

7.De Oliveira GS, Jr., Castro-Alves LJ, Nader A, Kendall MC, McCarthy RJ: Transversus abdominis plane block to ameliorate postoperative pain outcomes after laparoscopic surgery: a meta-analysis of randomized controlled trials. Anesthesia and analgesia 2014, 118(2):454-463.

8.Yu N, Long X, Lujan-Hernandez JR, Succar J, Xin X, Wang X: Transversus abdominis-plane block versus local anesthetic wound infiltration in lower abdominal surgery: a systematic review and meta-analysis of randomized controlled trials. BMC anesthesiology 2014, 14:121.

9.Brogi E, Kazan R, Cyr S, Giunta F, Hemmerling TM: Transversus abdominal plane block for postoperative analgesia: a systematic review and meta-analysis of randomized-controlled trials. Can J Anaesth 2016, 63(10):1184-1196.

10.McCrum CL, Ben-David B, Shin JJ, Wright VJ: Quadratus lumborum block provides improved immediate postoperative analgesia and decreased opioid use compared with a multimodal pain regimen following hip arthroscopy. Journal of hip preservation surgery 2018, 5(3):233-239.

11.Yousef NK: Quadratus Lumborum Block versus Transversus Abdominis Plane Block in Patients Undergoing Total Abdominal Hysterectomy: A Randomized Prospective Controlled Trial. Anesth Essays Res 2018, 12(3):742-747.

12.Oksuz G, Bilal B, Gurkan Y, Urfalioglu A, Arslan M, Gisi G, Oksuz H: Quadratus Lumborum Block Versus Transversus Abdominis Plane Block in Children Undergoing Low Abdominal Surgery: A Randomized Controlled Trial. Regional anesthesia and pain medicine 2017, 42(5):674-679.

13.Li G, Gai DX: Postoperative analgesia efficacy of quadratus lumborum block versus transversus abdominis plane block in patients undergoing caesarean section. International Journal of Anesthesiology and Resuscitation 2018, 39(4):338-340,345.

14.Blanco R, Ansari T, Riad W, Shetty N: Quadratus Lumborum Block Versus Transversus Abdominis Plane Block for Postoperative Pain After Cesarean Delivery: A Randomized Controlled Trial. Regional anesthesia and pain medicine 2016, 41(6):757-762.

15.Zhu MH, Tang Y, Xu Q, Qin Q, Chen Y: Quadratys lumborum block versus transversus abdominis plane block for analgesia after total abdominal hysterectomy. International Journal of Anesthesiology and Resuscitation 2018, 39(8):741-745. 
16.Kumar GD, Gnanasekar N, Kurhekar P, Prasad TK: A Comparative Study of Transversus Abdominis Plane Block versus Quadratus Lumborum Block for Postoperative Analgesia following Lower Abdominal Surgeries: A Prospective Double-blinded Study. Anesth Essays Res 2018, 12(4):919-923.

17.Han B, Wang WT, He AP: Comparison of ultrasound-guided quadratus lumborum block and transversus abdominis plane block combined with patient controlled intravenous analgesia with sufentani on post-operation analgesia after pendectomy. Journal of Clinical Anesthesiology 2017, 33(10):984-986.

18.Baytar C, Yilmaz C, Karasu D, Topal S: Comparison of Ultrasound-Guided Subcostal Transversus Abdominis Plane Block and Quadratus Lumborum Block in Laparoscopic Cholecystectomy: A Prospective, Randomized, Controlled Clinical Study. Pain Res Manag 2019, 2019:2815301.

19.Brennan F, Carr DB, Cousins M: Pain management: a fundamental human right. Anesthesia and analgesia 2007, 105(1):205-221.

20.Ueshima $\mathrm{H}$, Hiroshi O: Intermittent bilateral anterior sub-costal quadratus lumborum block for effective analgesia in lower abdominal surgery. Journal of clinical anesthesia 2017, 43:65.

21.Simpson JC, Bao X, Agarwala A: Pain Management in Enhanced Recovery after Surgery (ERAS) Protocols. Clinics in colon and rectal surgery 2019, 32(2):121-128.

22.Kehlet H, Dahl JB: Anaesthesia, surgery, and challenges in postoperative recovery. Lancet (London, England) 2003, 362(9399):1921-1928.

23.Gelman D, Gelmanas A, Urbanaite D, Tamosiunas R, Sadauskas S, Bilskiene D, Naudziunas A, Sirvinskas E, Benetis R, Macas A: Role of Multimodal Analgesia in the Evolving Enhanced Recovery after Surgery Pathways. Medicina (Kaunas, Lithuania) 2018, 54(2).

24.Rafi AN: Abdominal field block: a new approach via the lumbar triangle. Anaesthesia 2001, 56(10):1024-1026.

25.McDonnell JG, O'Donnell BD, Farrell T, Gough N, Tuite D, Power C, Laffey JG: Transversus abdominis plane block: a cadaveric and radiological evaluation. Regional anesthesia and pain medicine 2007, 32(5):399-404.

26. Hebbard P, Fujiwara Y, Shibata Y, Royse C: Ultrasound-guided transversus abdominis plane (TAP) block. Anaesthesia and intensive care 2007, 35(4):616-617.

27. Blanco R, Ansari T, Girgis E: Quadratus lumborum block for postoperative pain after caesarean section: A randomised controlled trial. European journal of anaesthesiology 2015, 32(11):812-818.

28.Sa M, Cardoso JM, Reis H, Esteves M, Sampaio J, Gouveia I, Carballada P, Pinheiro C, Machado D: [Quadratus lumborum block: are we aware of its side effects? A report of 2 cases]. Revista brasileira de 
anestesiologia 2018, 68(4):396-399.

29.Dhanjal S, Tonder S: Quadratus Lumborum Block. In: StatPearls. edn. Treasure Island (FL): StatPearls Publishing StatPearls Publishing LLC.; 2019.

30.Fusco P, Scimia P, Petrucci E, Di Carlo S, Paladini G, Marinangeli F: Transversus Abdominis Plane Block as Analgesic Technique for Postoperative Pain Management After Cesarean Section: No More? Regional anesthesia and pain medicine 2017, 42(4):541.

31.Willard FH, Vleeming A, Schuenke MD, Danneels L, Schleip R: The thoracolumbar fascia: anatomy, function and clinical considerations. Journal of anatomy 2012, 221(6):507-536.

\section{Tables}

Due to technical limitations, the tables could not be displayed here. Please see the supplementary files to access the tables.

\section{Figures}

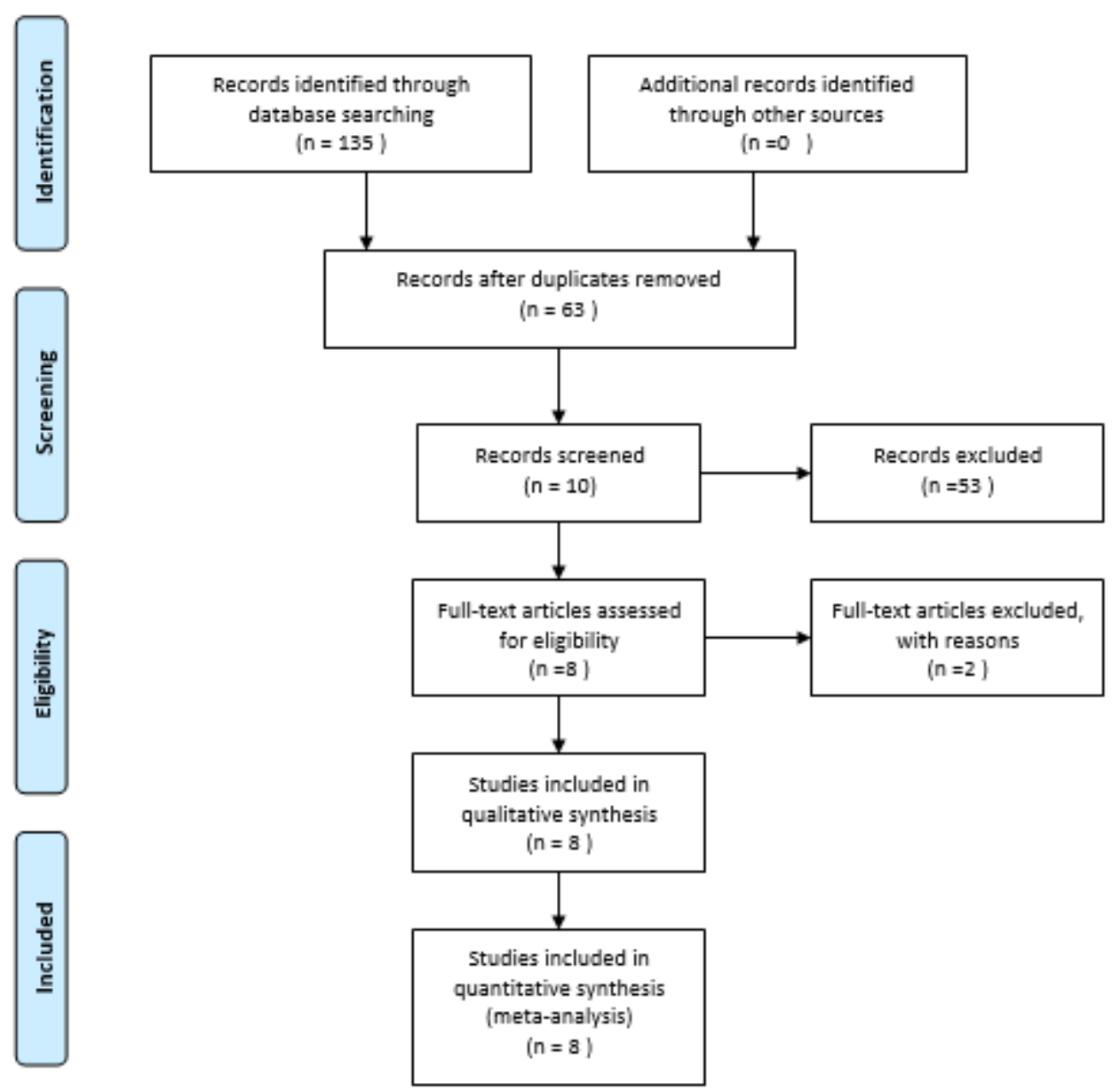

Figure 1 
PRISMA Flow Diagram

Random sequence generation (selection bias)

Allocation concealment (selection bias)

Blinding of participants and personnel (performance bias)

Blinding of outcome assessment (detection bias)

Incomplete outcome data (attrition bias)

Selective reporting (reporting bias)

Other bias
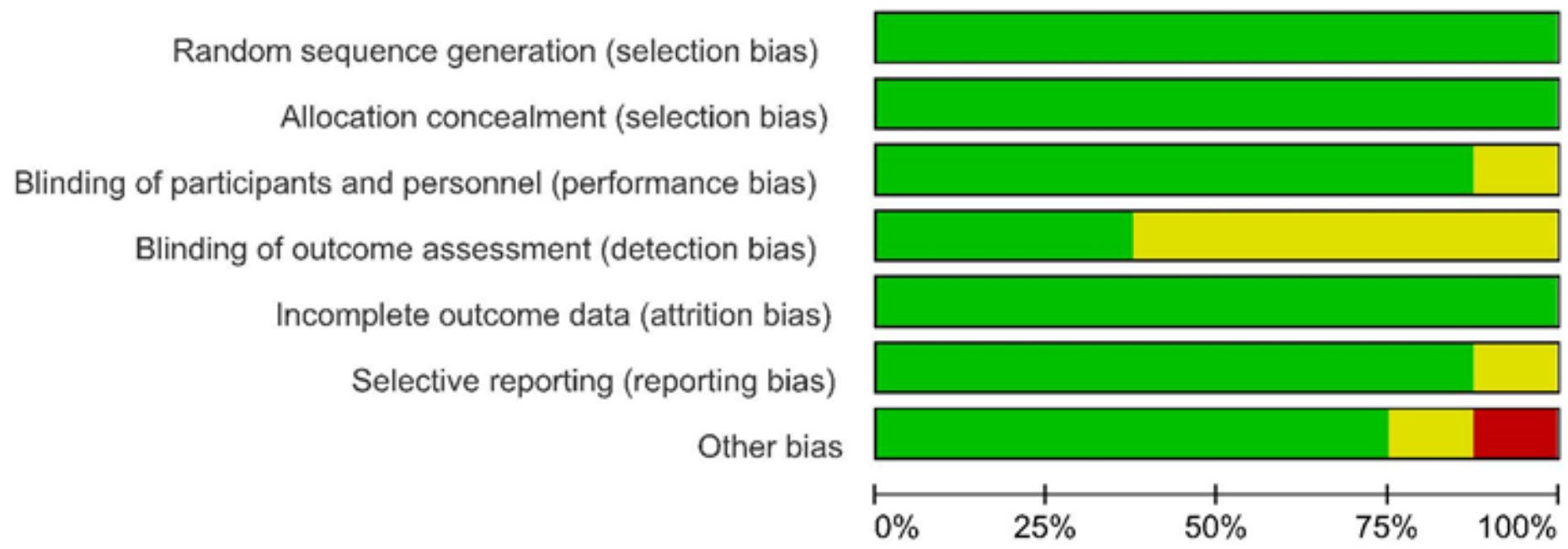

Figure 2

Risk of bias assessment of summary 


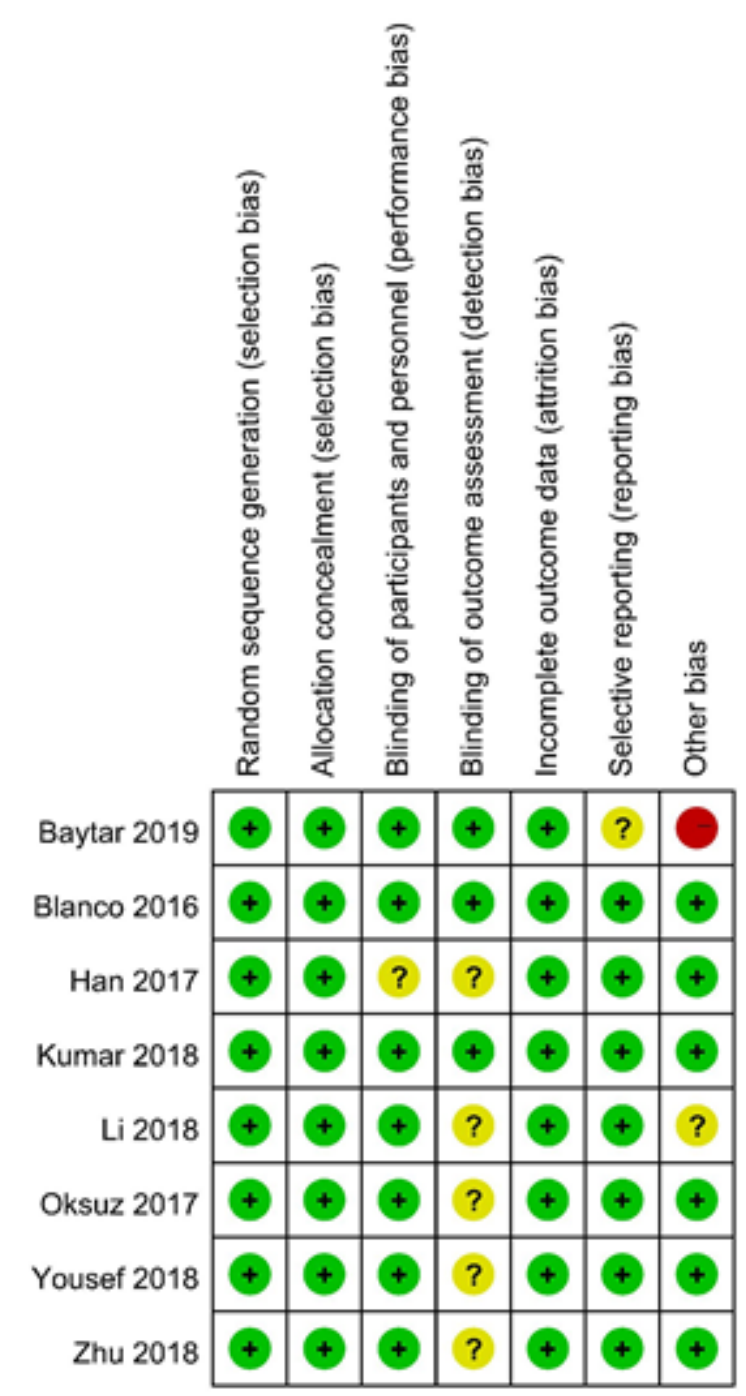

Figure 3

Risk of bias assessment of the studies 


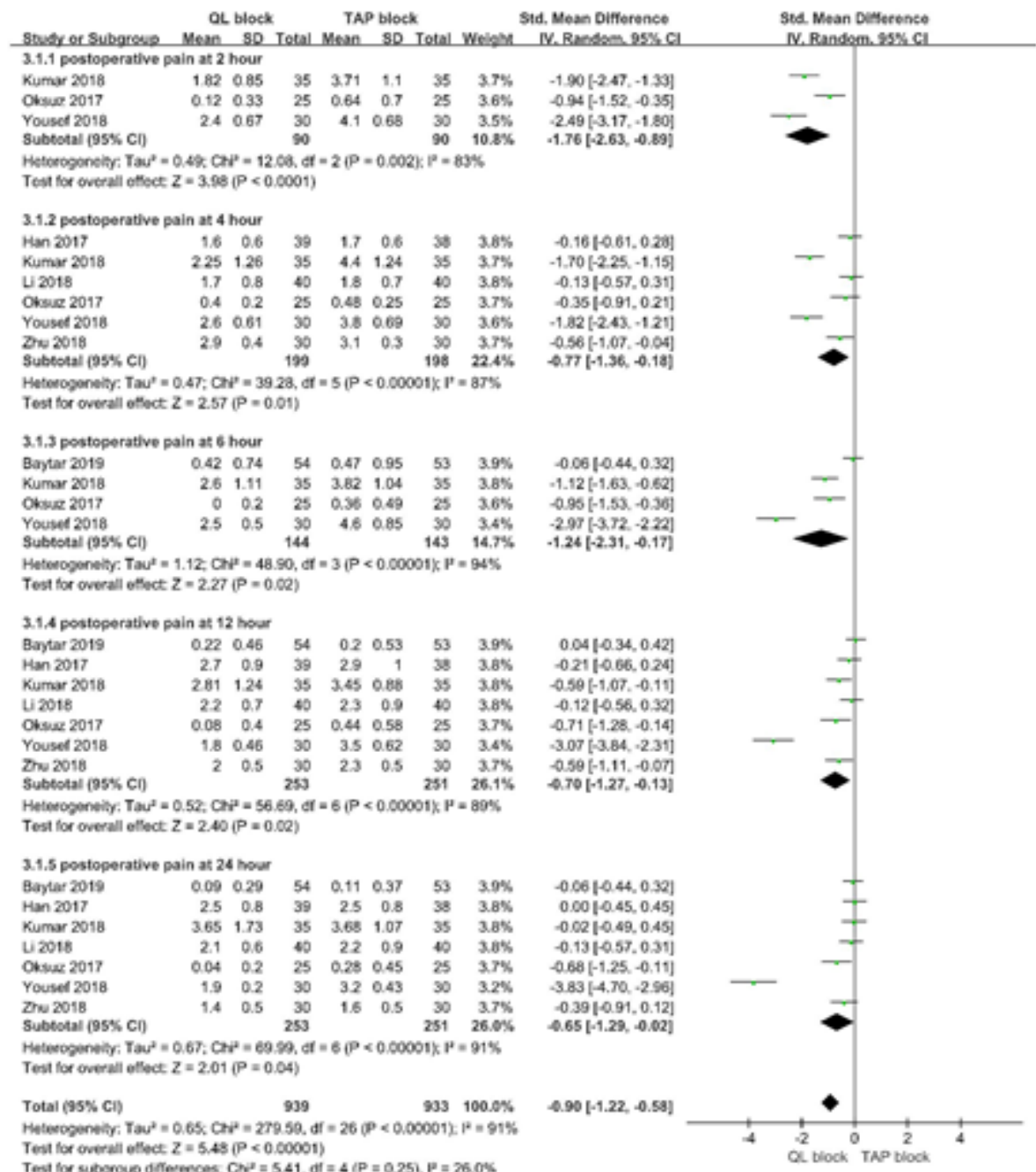

\section{Figure 4}

Forest plot for the meta-analysis of postoperative pain scores

\begin{tabular}{|c|c|c|c|c|c|c|c|c|c|c|c|c|}
\hline \multirow[b]{2}{*}{ Study or Subgroup } & \multicolumn{3}{|c|}{ QL block } & \multicolumn{3}{|c|}{ TAP block } & \multicolumn{2}{|r|}{ Std. Mean Difference } & \multirow{2}{*}{\multicolumn{4}{|c|}{$\begin{array}{l}\text { Std. Mean Difference } \\
\text { IV. Random. } 95 \% \mathrm{Cl}\end{array}$}} \\
\hline & Mean & SD & Total & Mean & SD & Total & Weight & IV. Random. $95 \% \mathrm{Cl}$ & & & & \\
\hline Blanco 2016 & 6 & 8.3 & 38 & 13.5 & 10.7 & 38 & $21.2 \%$ & $-0.78[-1.24,-0.31]$ & & - & & \\
\hline Han 2017 & 31.4 & 10.9 & 39 & 58.6 & 16.9 & 38 & $19.6 \%$ & $-1.90[-2.44,-1.36]$ & & - & & \\
\hline Kumar 2018 & 3.25 & 0.78 & 35 & 5.65 & 1.55 & 35 & $18.9 \%$ & $-1.93[-2.51,-1.36]$ & & \pm & & \\
\hline Li 2018 & 34.3 & 13.5 & 40 & 48.6 & 9.8 & 40 & $21.0 \%$ & $-1.20[-1.68,-0.72]$ & & $=$ & & \\
\hline Yousef 2018 & 10.06 & 3.8 & 30 & 14.46 & 3.4 & 30 & $19.3 \%$ & $-1.20[-1.76,-0.65]$ & & - & & \\
\hline Total $(95 \% \mathrm{Cl})$ & & & 182 & & & 181 & $100.0 \%$ & $-1.39[-1.83,-0.95]$ & & $\bullet$ & & \\
\hline $\begin{array}{l}\text { Heterogeneity: } \operatorname{Tau}^{2}= \\
\text { Test for overall effect: }\end{array}$ & $\begin{array}{l}0.18 ; \mathrm{Cr} \\
\mathrm{Z}=6.16\end{array}$ & $\begin{array}{l}i^{2}=14 \\
(P<C\end{array}$ & $.38, \mathrm{df}$ & $=4(P=$ & $=0.0$ & $; 1^{2}=7$ & $72 \%$ & & -10 & $-5 \quad 0$ & $\begin{array}{lr} & 5 \\
\text { TAP block }\end{array}$ & 10 \\
\hline
\end{tabular}

\section{Figure 5}

Forest plot for the meta-analysis of postoperative opioid consumption at 24 hours 


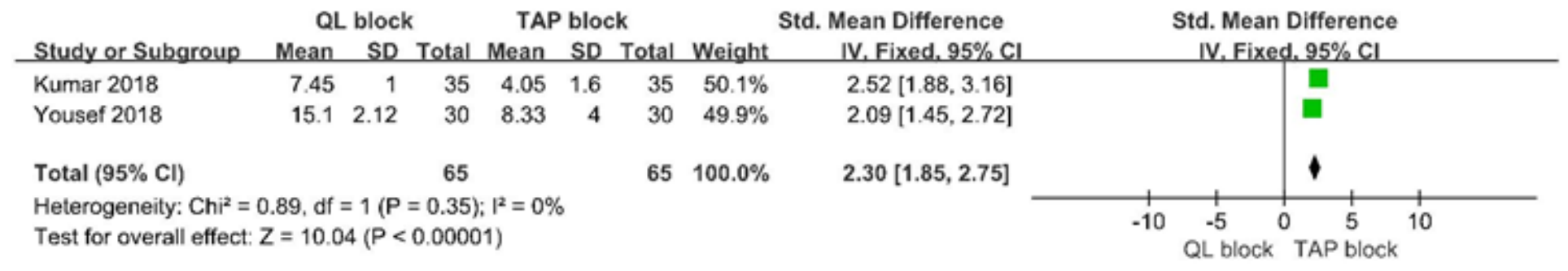

Figure 6

Forest plot for the meta-analysis of duration of postoperative analgesia

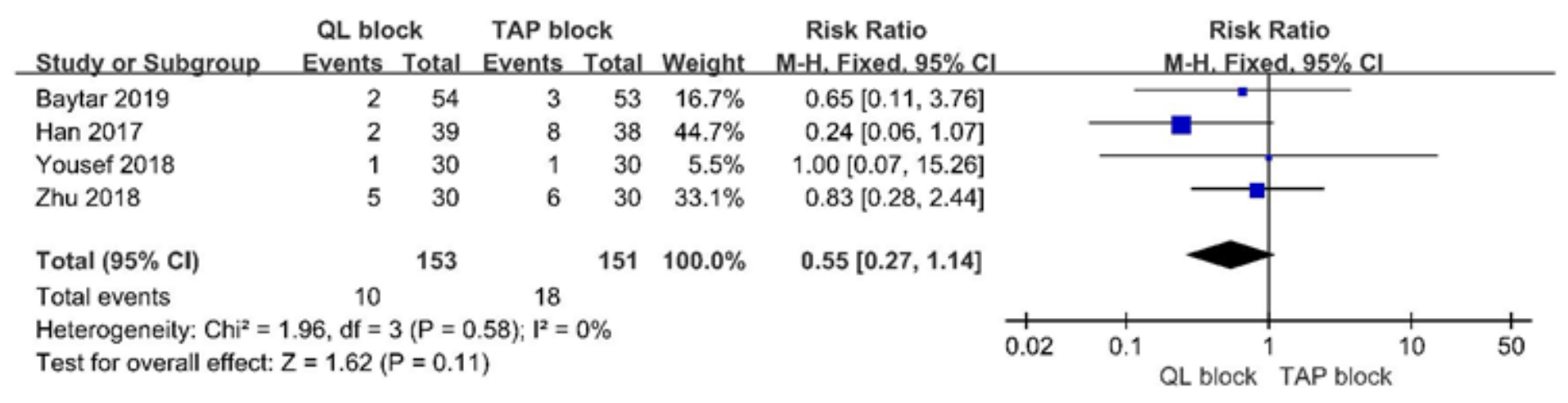

\section{Figure 7}

Forest plot for the meta-analysis of PONV

\section{Supplementary Files}

This is a list of supplementary files associated with this preprint. Click to download.

- PRISMA2009checklist.doc

- Table2.doc

- Table1.docx 